Nachhaltige Entwicklung als Freiheitsermöglichung

\section{Zum Spannungsverhältnis von Freiheit und Ökologie}

\author{
Wer im Sinne einer nachhaltigen Entwicklung Handlungsempfeh- \\ lungen gibt, sieht sich schnell in der Kritik, freiheitsfeindlich zu sein. \\ Wie können Wissenschaftler und Politiker mit dieser Kritik um- \\ gehen? Ein offensiver Gestaltungsdiskurs bietet vielversprechende \\ Ansätze. Von Reinhard Loske
}

$\mathbf{S}$ eit nunmehr knapp drei Jahrzehnten bemüht man sich in der nationalen und internationalen Debatte über nachhaltige Entwicklung relativ systematisch, die ökologische Frage mit der Gerechtigkeitsfrage zu verbinden. Die Aussage, dass innergesellschaftliche, internationale und intergenerative Gerechtigkeitsaspekte wesentliche Bestandteile jedweder Nachhaltigkeitsstrategie sein müssen und konsequent mit Betrachtungen zu den biophysikalischen Grenzen des Wachstums zu verknüpften sind, kann mindestens in der kritischen Nachhaltigkeitsforschung mittlerweile als Konsens gelten.

So systematisch wie mit der Gerechtigkeitsfrage hat sich die Nachhaltigkeitsforschung mit der Freiheitsfrage national wie international bisher nicht beschäftigt. Zwar tauchen Fragen danach, was denn Freiheit überhaupt sei und wie sie sich im Angesicht von ökologischen Grenzen neu interpretieren lasse, immer wieder auf, aber dass sich in dieser Sache bereits eine Art Konsens abzeichnet, kann man nicht behaupten.

\section{Zur Ökologie der Freiheit}

Die Zugänge zum Thema Freiheit und Ökologie können sehr unterschiedlich sein. So argumentiert Murray Bookchin, dass sich gesellschaftliche Hierarchien und ökologische Lebensführung ausschließen. Er plädiert deshalb für eine dezentralisierte Gesellschaft, in der Selbstverwaltung, Selbstorganisation und direkte Demokratie eine starke Ge- genmacht zu Staats- und Kapitalinteressen bilden (Bookchin 1985). Die aktuellen Diskussionen über ökologische Verantwortung in der Bürgergesellschaft können hier nahtlos anknüpfen (Loske

Tine Stein sieht aus einer eher wertkonservativen Position schauend „Demokratie und Verfassung an den Grenzen des Wachstums“ und empfiehlt der Gesellschaft eine moderne Odysseus-Interpretation (Stein 1998). Dieser Held der griechischen Mythologie habe sich dem verlockenden, aber auch in den Untergang führenden Gesang der Sirenen („Du kannst von allem immer mehr haben! Also konsumiere!") dadurch widersetzt, dass er sich aus besserer Einsicht heraus selbst bindeen ließ. Hier erscheint die vernunftgesteuerte Selbstbegrenzung als höchste Form der Freiheit. Reduktionsziele für Klimagase, Ressourcen-, Energie- und Flächenverbrauch sind in dieser Logik Ausdrucksformen einer vom falschen Fortschrittsglauben befreiten Gesellschaft.

Man könnte auch bei Rosa Luxemburg und ihrem berühmten Zitat anknüpfen, dass die eigene Freiheit dort endet, wo diejenige der anderen eingeschränkt wird, der Autofahrer also durchaus auch die Interessen der Straßenanwohner, der Radfahrer und Fußgänger zu berücksichtigen hat, etwa indem er Abgasnormen erfüllt oder Tempolimits einhält. Bei jeder potenziell umweltschädigenden Aktivität, etwa der Verbrennung von Erdöl, stellt sich natürlich die Frage, wer die Anderen sind, de2013). ren Freiheitsrechte man zu beachten hat. Sind es auch die zukünftigen Generationen, die Menschen in anderen Teilen der Welt, die Tiere und Pflanzen?

\section{Auf dem Weg zur Ökodiktatur?}

Der Spielraum für Antworten auf diese Frage ist sehr groß. Und so verwundert es auch nicht, dass es in pluralistischen Gesellschaften tatsächlich sehr heterogene Antworten gibt. Die Debatte über den freiwilligen Veggie Day, einen Vorschlag im grünen Bundestagswahlprogramm 2013, der im Grunde an Harmlosigkeit kaum zu überbieten ist, hat hier leider ein bezeichnendes Licht auf die öffentliche Diskussionskultur in unserem Lande geworfen. Es reichen ein, zwei Hetzartikel in der Zeitung mit den großen Buchstaben. Und schon bricht auch in den seriösen Medien eine Debatte los, in der man den Eindruck gewinnen kann, wir stünden kurz vor dem Einstieg in die Ökodiktatur. Und jetzt will uns die Europäische Union sogar noch energieeffiziente Staubsauger vorschreiben. Ungeheuerlich (siehe z. B. FAZ 2014)!

Das Ziel der heftigen Attacken auf die Grünen, die auch sogleich Besserung gelobten und nun niemandem mehr die Wurst wegnehmen wollen, liegt auf der Hand. Keiner soll mehr ungestraft das politisch verminte Gelände der Lebensstilfragen oder gar der Konsumkritik betreten. Wer es dennoch wagt, demaskiert sich selbst als übler Freiheitsfeind, dem es Einhalt zu gebieten gilt.

Wie kann in Forschung, Politik und gesellschaftlicher Debatte mit dieser medial geschürten und in bestimmten Teilen der Gesellschaft auch gern aufgenommenen Stimmung umgegangen werden? Nun, Patentrezepte gibt es sicher nicht, aber vier Grundorientierungen scheinen mir sinnvoll:

I Zunächst geht es um Selbstbehauptung. Ja, Regierung bedeutet immer auch Regulierung. Die Scheidelinie verläuft im Regelfall nicht zwischen Regulierung und Nichtregulierung, sondern zwischen guter und schlech- 
ter, angemessener und unangemessener Regulierung. Es kann also in der Tat Über- wie Unterregulierung geben. Darüber muss immer wieder neu und im Einzelfall entschieden werden.

- Man sollte sich bewusst sein, dass es machtvolle Kreise gibt, die den Sack schlagen, aber den Esel meinen, die vermeintlichen Öko-Dirigismus anprangern, aber in Wahrheit Probleme wie die Ressourcenübernutzung, den Schwund biologischer Vielfalt oder den Klimawandel in Abrede stellen. Die Auseinandersetzung mit diesem Kreis von Personen und Interessengruppen muss transparent und für andere nachvollziehbar sein.

- Bei der Wahl von angemessenen Instrumenten für eine Politik der Nachhaltigkeit muss der Aspekt der größtmöglichen Freiheit bei der Erreichung der demokratisch bestimmten Ziele immer mitgedacht werden. Als Leitorientierung kann gelten: Wo Gefahr im Verzug ist, muss das Ordnungsrecht mit seinen Ge- und Verboten greifen. Wo ökologisch unverträgliche Mengenprobleme vorliegen, etwa beim Verbrauch von Energie, Rohstoffen oder Flächen sowie beim Ausstoß klimaverändernder Spurengase, sind besonders ökonomische Instrumente wie die Ökosteuer oder der Emissionshandel geeignet, um der Innovationsdynamik eine neue Richtung zu geben. Wo es um den dauerhaften Wandel von Werten, Lebensstilen und sozialer Praxis geht, sind Bildung, Erziehung, Information und gesellschaftliche Anerkennung für vorbildliches Verhalten die Mittel der Wahl. Auch hier gilt: Darüber muss immer wieder gesprochen werden.

- Intelligente Nachhaltigkeitspolitik versucht den Freiheitsbegriff glaubwürdig für sich zu reklamieren und Bedingungen für freiheitliches Handeln zu schaffen. Das reicht von der Freiheit, sich unbehelligt von Beeinträchtigungen wie Lärm und Abfällen in der freien Natur bewegen zu können, über die freien Gemeinschaften, die sich aus freien Stücken zusam- menschließen, um Stadtgärten und Tauschringe, Reparaturcafés und Recyclingbörsen, Energiegenossenschaften oder Erzeuger-VerbraucherGemeinschaften ins Leben zu rufen (Loske 2014), bis zu den Unternehmen, die sich frei dazu entscheiden, bei der Bewältigung der ökologischen Krisen nicht Teil des Problems, sondern Teil der Lösung zu sein - und damit idealerweise sogar gutes Geld verdienen.

Die Protagonisten der Nachhaltigkeit sollten sich in Zukunft verstärkt darum bemühen, ihre Argumentation von einem defensiven Abwehrdiskurs („Wir sind doch gar nicht so freiheitsfeindlich, wie behauptet!“) in einen offensiven Gestaltungsdiskurs zu überführen, in dem die Bewahrung der natürlichen Lebensgrundlagen als Freiheitsermöglichung für heutige und zukünftige Menschen erkennbar wird.

\section{Literatur}

Bookchin, M. (1985): Die Ökologie der Freiheit. Wir brauchen keine Hierarchien. Weinheim/ Basel, Beltz.

FAZ vom 23. 5. 2014, abrufbar unter: http:// www.faz.net/aktuell/politik/europaeischeunion/eu-energiepolitik-wut-ueberregelungswut-12955017.html (abgerufen am 5. 10. 2014).

Loske, R. (2013): Ökologische Verantwortung in der Bürgergesellschaft. In: Töpfer, K. et al. (Hrsg.): Verändern durch Wissen. Chancen und Herausforderungen demokratischer Beteiligung. München, Oekom. S. 19-29.

Loske, R. (2014): Neue Formen kooperativen Wirtschaftens als Beitrag zur nachhaltigen Entwicklung. Leviathan 42/3. S. 463-485.

Stein, T. (1998): Demokratie und Verfassung an den Grenzen des Wachstums. Zur ökologischen Kritik und Reform des Verfassungsstaates. Opladen, Westdeutscher Verlag.

AUTOR + KONTAKT

Dr. Reinhard Loske ist Professor für Politik, Nachhaltigkeit und Transformationsdynamik an der Universität Witten/Herdecke und Senior Associate Fellow der Deutschen Gesellschaft für Auswärtige Politik in Berlin.

Dr. Reinhard Loske, Universität Witten/Herdecke, Alfred-Herrhausen-Str. 50, 58448 Witten. E-Mail: reinhard.loske@uni-wh.de, Website: www.loske.de

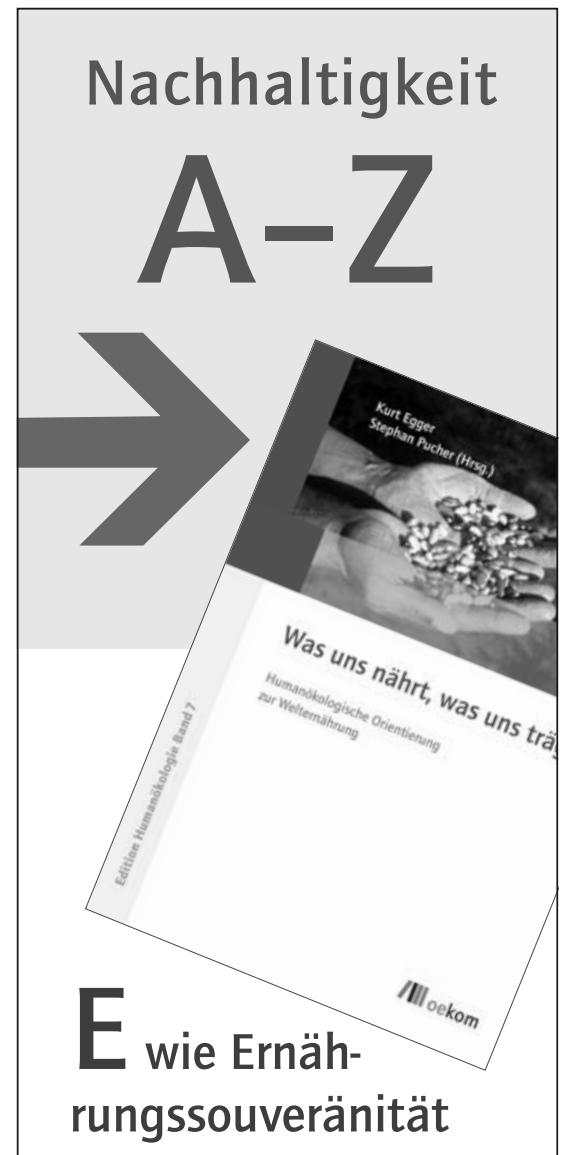

Bei Ernährung denken wir zuerst ans Essen. Dies greift jedoch vor dem Hintergrund ökologischer und sozialer Herausforderungen oft viel zu kurz. Die Autor(inn)en nehmen daher die emotionale Seite der Nahrungsaufnahme ebenso wie die sozialen Aspekte der Ernährung in den Blick. Beispielhaft zeigen sie anhand kleinbäuerlicher (Bio-)Landwirtschaft in den Tropen Lösungswege aus der globalen Ernährungskrise auf.

K. Egger, S. Pucher (Hrsg.)

Was uns nährt, was uns trägt

Humanökologische Orientierung zur Welternährung

Edition Humanökologie Band 7

312 Seiten, broschiert, 39,95 Euro, ISBN 978-3-86581-319-0

Erhältlich bei

www.oekom.de

oekom@verlegerdienst.de

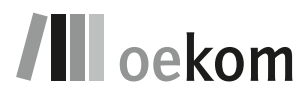

Die guten Seiten der Zukunft 